\title{
Homozygous SMN2 deletion is a protective factor in the Swedish ALS population
}

\author{
Philippe Corcia ${ }^{\star, 1,2}$, Caroline Ingre ${ }^{4,5}$, Helene Blasco ${ }^{2,3}$, Rayomand Press ${ }^{5}$, Julien Praline ${ }^{1,2}$, Catherine Antar ${ }^{2,3}$, \\ Charlotte Veyrat-Durebex ${ }^{2,3}$, Yves-Olivier Guettard ${ }^{2}$, William Camu ${ }^{6}$, Peter M Andersen ${ }^{4}$, Patrick Vourc' ${ }^{2,3}$ \\ and Christian R Andres ${ }^{2,3}$
}

\begin{abstract}
Abnormal survival motor neuron 1 (SMN1)-copy number has been associated with an increased risk of amyotrophic lateral sclerosis (ALS) in French and Dutch population studies. The aim of this study was to determine whether SMN gene copy number increases the risk of ALS or modulates its phenotype in a cohort of Swedish sporadic ALS (SALS) patients. In all, 502 Swedes with SALS and 502 Swedish controls matched for gender and age were enrolled. SMN1 and SMN2 gene copy numbers were studied by a semiquantitative PCR method. A genotype-phenotype comparison was performed in order to determine whether $S M N$ genes modulate the phenotype of ALS. The results were also compared with our previously reported French cohort of ALS patients. There was no difference between Swedish patients and controls in the frequency of SMN1 and SMN2 copy numbers. The frequency of SMN1 gene copies differed significantly between the French and Swedish ALS populations. The duration of the disease was significantly longer in the Swedish cohort with homozygous deletions of SMN2 when compared with the French cohort. Abnormal SMN1 gene copy number cannot be considered as a universal genetic susceptibility factor for SALS and this result underlines the importance of reproducing association gene studies in groups from different origins. We also suggest that SMN2 gene copy number might have different effects on ALS progression in disparate human populations.
\end{abstract}

European Journal of Human Genetics (2012) 20, 588-591; doi:10.1038/ejhg.2011.255; published online 25 January 2012

Keywords: SMN1 gene; SMN2 gene; sporadic amyotrophic lateral sclerosis; association studies

\section{INTRODUCTION}

Amyotrophic lateral sclerosis (ALS) is a fatal neurodegenerative disorder described worldwide. ${ }^{1}$ During the last 10 years, an extensive literature has focused on genetic susceptibility factors in populations from different origins. Currently, there is no universal genetic factor associated with the majority of ALS cases.

A series of studies performed independently in French and Dutch ALS populations suggests that an abnormal survival motor neuron 1 (SMN1) gene copy number is associated with increased risk of ALS $(\mathrm{OR}=2.8)^{2-4}$

These studies relied principally on the following facts: homozygous deletion of the SMN1 gene causes spinal muscular atrophy (SMA), another motor neuron disease and SMN2 gene copy number modulates the phenotype of SMA. ${ }^{5}$ SMN genes encode a protein important for the formation of spliceosomal small nuclear ribonucleoprotein particles, which may modulate longevity of motor neurons. ${ }^{6}$

We analyzed SMN1 and SMN2 copy numbers in a large welldefined population of Swedish patients diagnosed with sporadic ALS (SALS) to determine whether copy number in the SMN genes modulates the risk of ALS or modifies the phenotype and prognosis of SALS patients. We also compared the SMN-genotype distribution in the Swedish population to that in a similar French population.

\section{MATERIALS AND METHODS}

Study population

Clinical data and blood samples, collected between 1996 and 2008, were obtained from 502 SALS patients and 502 controls. The participants signed consent forms approved by Medical Ethical Review Boards in Sweden that adhere to the principles described in the Declaration of Helsinki. All subjects were of Swedish origin dating at least three generations. To maintain comparability between groups, patients and controls living in Sweden, but of other ethnicity, were excluded from the study. Three genes have been shown to be associated with rare forms of familial ALS: SOD1, FUS and TDP43; as this study enrolled patients with SALS, only those subjects who did not have mutations in one of these genes were enrolled.

The controls were matched to cases for age, sex and ethnicity. All patients met the El Escorial World Federation criteria for the diagnosis of definite or probable ALS. ${ }^{8}$ We collected clinical data comprising gender, age and site of onset of first motor symptom (spinal or bulbar), and duration of ALS (the interval between the first symptoms and death or tracheotomy).

The results of the Swedish population were compared with our French ALS cohort of 600 SALS patients, for whom we previously reported an association with SMN1 gene copy numbers. ${ }^{2}$ We first compared the ALS phenotype (gender, site of onset, age of onset and duration of the disease) and the SMN1 and SMN2 copy numbers frequency in Swedish patients to that of our French ALS and controls groups. ${ }^{2}$

Experimental protocol

Genomic DNA was extracted by standard procedures from peripheral blood. $S M N$ genes copy numbers were determined by a quantitative multiplex PCR

${ }^{1}$ Centre SLA, CHRU de Tours, Tours, France; ${ }^{2}$ UMR INSERM U930, CNRS 3106, Université François Rabelais de Tours, Tours, France; ${ }^{3}$ Laboratoire de Biochimie et Biologie moléculaire, Hôpital Bretonneau, CHRU de Tours, Tours, France; ${ }^{4}$ Department of Clinical Neuroscience, Umeå University, Umeå, Sweden; ${ }^{5}$ Department of Neurology, Karolinska University hospital, Stockholm, Sweden; ${ }^{6}$ Centre SLA, CHU de Montpellier, Université Montpellier 1, Montpellier, France

${ }^{*}$ Correspondence: Professor P Corcia, UMR INSERM U930, Faculté de Médecine, Université François Rabelais de Tours, 10, Boulevard Tonnellé, 37044 Tours CEDEX1, France. Tel: +33247473724; Fax: +33247473808; E-mail: corcia@med.univ-tours.fr

Received 3 August 2011; revised 17 November 2011; accepted 7 December 2011; published online 25 January 2012 
technique as previously described. ${ }^{3}$ Data were analysed using the Genescan software (Perkin Elmer, Courtaboeuf, France). The SMN1 and SMN2 gene copy numbers were calculated using the ratio SMN1/CFTR (Cystic fibrosis transmembrane conductance regulator gene) and SMN2/CFTR of each peak area. Analysis of $S M N$ genes copy number was performed by an investigator blinded to disease status (ALS or control).

\section{Statistical analysis}

All analysis was performed with JMP statistical software version 7.0.2 (SAS Institute, Cary, NC, USA).

We first analysed associations between $S M N 1$ or SMN2 copy numbers and the presence of SALS within the Swedish population using a $\chi^{2}$-test. A correction for multiple tests was applied to adjust the $P$ values by accounting for the two loci (SMN1 and SMN2 copy numbers) analysed: significance thresholds were taken as 0.025 .

In a second step, we compared gender, site of onset, age of onset and SMN1 as well as SMN2 copy numbers in Swedish and French patients using a $\chi^{2}$ or $t$-test. Based on a violation of the assumption of proportional hazards, the Wilcoxon's test, placing more weight on early survival times, was used to compare survival curves between both groups.

We then focused survival analysis on the subgroups of patients from France and Sweden with homozygous SMN2 deletion and SMN1 abnormal gene copy numbers. We evaluated all parameters previously described. The different factors having a significant influence on survival analyzed using Kaplan-Meier methods were further explored with multivariate Cox proportional hazard models.

\section{RESULTS}

A total of 216 women and 286 men with SALS were included in the study. In all, $173(34.5 \%)$ patients had bulbar onset, $144(28.7 \%)$ had upper limb and 127 (25.3\%) lower limb. The remaining 58 (11.5\%) had spinal onset either in thoracic myotomes or in more than one limb. Mean age of onset was $61.8 \pm 13.4$ years for the 463 patients from whom reliable data were available. Median survival time was 32.7 months (95\% CI 29.6-34.8 months) ( $n=463)$.

Results of the SMN1 and SMN2 copy number analysis are summarized in Table 1. In the Swedish sample, there was no

Table 1 Frequency of SMN1 and SMN2 copy number in ALS and control groups from France and Sweden

\begin{tabular}{|c|c|c|c|c|}
\hline & \multicolumn{2}{|c|}{ ALS population } & \multicolumn{2}{|c|}{ Control group } \\
\hline & Sweden & France & Sweden & France \\
\hline \multicolumn{5}{|c|}{ SMN1 copy number } \\
\hline 1 SMN1 & $16(3.2 \%)$ & $36(6.0 \%)$ & $9(1.8 \%)$ & $13(2.1 \%)$ \\
\hline 2 SMN1 & 467 (93.0\%) & $529(88.2 \%)$ & 479 (95.4\%) & $593(95.5 \%)$ \\
\hline 3 SMN1 & $19(3.8 \%)$ & $35(5.8 \%)$ & $14(2.8 \%)$ & $15(2.4 \%)$ \\
\hline Total & 502 & 600 & 502 & 621 \\
\hline$P$-Value & $0.24^{a}$ & & & \\
\hline$P$-Value & $0.02^{b}$ & & $0.87^{b}$ & \\
\hline \multicolumn{5}{|c|}{ SMN2 copy number } \\
\hline O SMN2 & 29 (5.8\%) & $54(9.0 \%)$ & $37(7.4 \%)$ & 52 (8.4\%) \\
\hline 1 SMN2 & $189(37.6 \%)$ & 234 (39.0\%) & $212(42.2 \%)$ & 239 (38.4\%) \\
\hline 2 SMN2 & 276 (55.0\%) & $305(50.8 \%)$ & 247 (49.2\%) & $321(51.7 \%)$ \\
\hline 3 SMN2 & $8(1.6 \%)$ & $7(1.2 \%)$ & $6(1.2 \%)$ & $9(1.5 \%)$ \\
\hline Total & 502 & 600 & 502 & 621 \\
\hline$P$-Value & $0.23^{a}$ & & & \\
\hline$P$-Value & $0.15^{\mathrm{b}}$ & & $0.61^{b}$ & \\
\hline
\end{tabular}

Abbreviations: ALS, amyotrophic lateral sclerosis; SMN, survival motor neuron. ${ }^{2}$ Comparison between both Swedish groups.

b'Comparison between the French and the Swedish groups. significant difference in the frequency of SMN1 or SMN2 copy number between cases and controls $(P=0.24$ and $P=0.23$, respectively). A significant difference in SMN1 copy number was found between the Swedish and French SALS populations $(P=0.02)$, and no difference between the Swedish and French control groups $\left(\chi^{2}\right.$-test, $\left.P=0.87\right)$.

There was no difference between both ALS groups in the age of onset $(P=0.23)$, gender $(P=0.90)$, the site of onset, and disease duration (respectively, $\chi^{2}$-test, $P=0.38$, and Wilcoxon's test: $P=0.09$; Table 2).

No phenotypic difference was observed between Swedish and French ALS sample according to SMN1 copy number.

As homozygous SMN2 deletion has also been linked to motor neuron disorders (MNDs), either through an increased frequency in adult sporadic lower motor neuron diseases or a shorter duration of disease in Dutch patients with ALS, we concluded our analysis by focusing on the subgroup of patients from France and Sweden with homozygous SMN2 deletions to determine whether this genotype was associated with a particular phenotype. ${ }^{9-11}$

Although non-significant, the frequency of homozygous SMN2 deletion was lower in the Swedish than in the French ALS patients (5.8 vs 9.0\%, $P=0.15$, Table 1). Genotype-Phenotype correlation analysis showed that survival was longer in Swedish patients $(n=27)$ than French patients $(n=50)$ with homozygous SMN2 deletion (37.6 (27.0-46.4) months vs 27.0 (19.1-32.1) months, $P=0.039$, Table 3, Figure 1)

Site of onset and age of onset were significant predictors of survival within subgroups defined by the SMN1 or SMN2 gene copy numbers (data not shown), but gender had no effect $(P=0.40)$. In a multivariate Cox proportional hazard model, including site of onset and country of origin, these variables remained significant determinants of overall survival within the subgroup of ALS patients having homozygous SMN2 deletion. The hazard ratios were $1.7(1.1-2.8)(P=0.038)$ for country of origin and $2.5(1.5-4.2)(P<0.001)$ for site of onset (Table 4).

Table 2 Characteristics of the French and Swedish ALS population

\begin{tabular}{|c|c|c|c|}
\hline Characteristics & France & Sweden & P-Value \\
\hline \multicolumn{4}{|c|}{ Age of onset (years) } \\
\hline Mean & 60.9 & 61.8 & 0.23 \\
\hline SD & 12.7 & 13.4 & \\
\hline $\mathrm{N}$ & 585 & 463 & \\
\hline \multicolumn{4}{|l|}{ Gender, n (\%) } \\
\hline Male & $344(57.3 \%)$ & $286(56.9 \%)$ & 0.90 \\
\hline Female & $256(42.7 \%)$ & $216(43.1 \%)$ & \\
\hline \multicolumn{4}{|l|}{ Site of onset } \\
\hline Bulbar & $188(31.3 \%)$ & $173(34.5 \%)$ & 0.38 \\
\hline UL & $95(15.9 \%)$ & $144(28.7 \%)$ & \\
\hline LL & $107(17.8 \%)$ & $127(25.3 \%)$ & \\
\hline Spinal & $210(35.0 \%)$ & $58(11.5 \%)$ & \\
\hline \multicolumn{4}{|c|}{ Duration of disease (months) } \\
\hline Median & 29.5 & 32.7 & 0.09 \\
\hline $95 \% \mathrm{Cl}$ & 28.0-31.1 & $29.6-34.8$ & \\
\hline $\mathrm{N}$ & 512 & 463 & \\
\hline
\end{tabular}

Abbreviation: ALS, amyotrophic lateral sclerosis. 
Table 3 Duration of ALS according to SMN2 copy number in French and Swedish populations

\begin{tabular}{lccc}
\hline $\begin{array}{l}\text { Number of } \\
\text { SMN2 copy }\end{array}$ & $\begin{array}{c}\text { French ALS population } \\
\text { (months) (n) }\end{array}$ & $\begin{array}{c}\text { Swedish ALS population } \\
\text { (months) (n) }\end{array}$ & P-Value \\
\hline $0(n=77)$ & $(27.0(19.1-32.1))(50)$ & $(37.6(27.0-46.4))(27)$ & 0.039 \\
$1(n=374)$ & $(31.0(28.0-33.0))(199)$ & $(34.7(29.0-37.5))(175)$ & 0.407 \\
$2(n=510)$ & $(29.0(27.0-32.0))(261)$ & $(31.0(27.5-33.0))(249)$ & 0.653 \\
$3(n=12)$ & $(27.0(17.0-37.0))(6)$ & $(69.5(42.0-N A))(6)$ & NA \\
\hline
\end{tabular}

Abbreviations: ALS, amyotrophic lateral sclerosis; NA, not applicable; SMN, survival motor neuron.

For duration, values were medians (Cl 95\%).

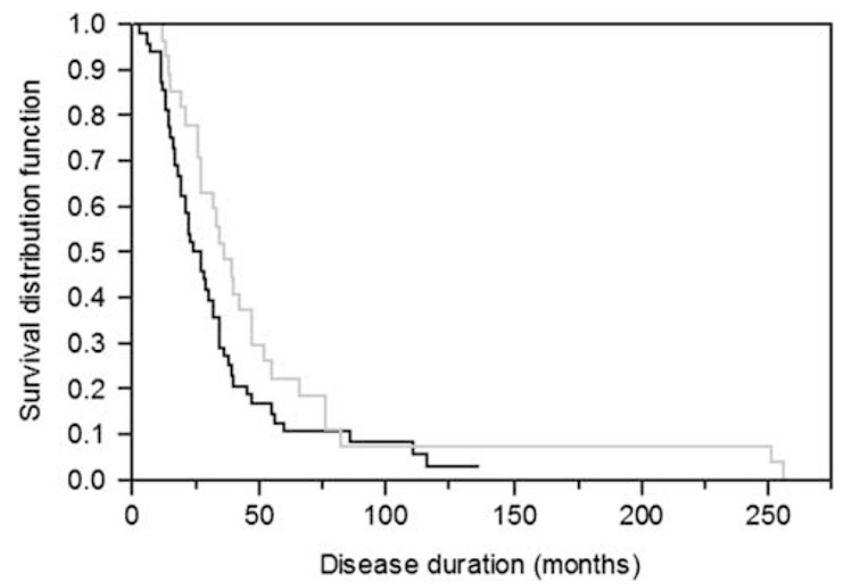

Figure 1 Kaplan-Meier curves representing overall survival of ALS patients having homozygous deletion for $S M N 2$ gene $(P=0.039)$. Black curves correspond to French ALS patients $(n=50)$ and grey curves correspond to Swedish ALS patients ( $n=27$ ).

Table 4 Cox proportional hazards model including site of onset (bulbar or spinal) and origin (France or Sweden) within the subgroup of patients having homozygous deletion for SMN2 gene $(n=77)$

\begin{tabular}{lcccc}
\hline & $\begin{array}{c}\text { Number of } \\
\text { patients }\end{array}$ & $\begin{array}{c}\text { Disease duration (months) } \\
\text { Median (Cl 95\%) }\end{array}$ & $\begin{array}{c}\text { Hazard ratio } \\
(95 \% \mathrm{Cl})\end{array}$ & P-Value \\
\hline $\begin{array}{l}\text { Site of onset } \\
\text { Bulbar }\end{array}$ & $n=31$ & $25.7(19.0-33.1)$ & $2.5(1.5-4.2)$ & $<0.001$ \\
Spinal & $n=46$ & $34.1(27.0-47.0)$ & & \\
& & & & \\
Origin & & & & \\
French & $n=50$ & $27.0(19.2-32.1)$ & $1.7(1.1-2.8)$ & 0.038 \\
Sweden & $n=27$ & $36.2(26.0-47.2)$ & & \\
\hline
\end{tabular}

Abbreviations: $\mathrm{Cl}$, confidence interval; SMN, survival motor neuron.

Two Swedish ALS patients with respiratory onset were excluded of this study.

\section{DISCUSSION}

An emerging way of research in neurodegenerative disorders relied on the theory of copy number variant: this was described in hereditary sensory motor neuropathies, Alzheimer's and Parkinson's diseases with abnormal number of copies of the PMP-22 gene, APP gene and synuclein genes, respectively. ${ }^{12-14}$ The association between SMN1 gene copy number and ALS previously found in French and Dutch population corroborated this hypothesis in MND. ${ }^{2-4}$ As almost all genetic association studies led to controversial results, we aimed to strengthen the findings of an association between SMN1 copy number and SALS in a third unrelated population.

SMN1 and SMN2 copy numbers were analysed blindly in a sample of Swedish ALS patients according to a quantitative analysis method we previously reported in French patients. ${ }^{3}$ We did not find differences in the frequency of SMN1 and SMN2 copy numbers between Swedish ALS patients and controls. Because of the incidence of SMA (homozygous deletion of SMN1) in the Swedish population is around 1 in 12000 live births, ${ }^{15}$ the frequency of heterozygous SMN1 deletions in Sweden can be estimated to be around $1 / 55$, similar to what has been reported in France. ${ }^{16}$ The frequency of heterozygous SMN1 deletions found in the Swedish population in this study was in accordance with those reports, suggesting that our control population was representative of the Swedish population as a whole. These lack of association between ALS and SMN1 gene copy number is in contrast to results in French and Dutch cohorts in which abnormal copy numbers of SMN1 have been associated with an increased risk of ALS. ${ }^{2-4}$

Although the management of ALS was similar in both countries all along the period of collection of samples, there was a significant difference between the French and the Swedish ALS cohorts in the frequency of SMN1 copy number. Abnormal SMN1 gene copy number, therefore, cannot be considered as a universal genetic susceptibility factor for SALS.

Although the difference did not reach significance, the frequency of SMN2 homozygous deletions was lower in the Swedish than in the French ALS group. Disease duration was longer in Swedish patients with homozygous deletions in SMN2 when compared with the French cohort and this was not the case for one and two SMN2 copies. We did not include in our analysis the use or not of invasive ventilation or gastronomy because of the absence of formal evidence of an impact of these supports on survival. ${ }^{17}$ This result led us to propose that a genetic or an environmental factor might modulate the effect of homozygous SMN2 deletions on survival. The survival curves for French and Swedish ALS populations with homozygous SMN2 deletions crossed late, possibly because of a high number of French ALS lost to follow-up after 75 months and to a long survival in Swedish ALS patients, not related to different management of the disease. There was no such bias during the 75 first months of evolution that might lead to differences in survival between groups. This risk of false positive results due to differences in disease duration in the two samples was corrected with the Wilcoxon's test, which gives more weight to deaths at early time points.

These data indicate that ALS populations cannot be considered one homogenous group and that genetic factors may be specific to given populations. A similar pattern of variable gene distribution has been found among ALS patients with mutations in causative genes for familial ALS: the D90A SOD1 mutation predominates in northern Sweden and Finland, and the L144F in eastern Mediterranean countries (Italy and Istro-Rumania). ${ }^{18,19}$ SOD1 gene mutations are rare in The Netherlands and Denmark. ${ }^{20,21}$ Mutations in the Angiogenin gene are rare in Sweden, England and Germany, but relatively common in Scotland and Ireland. ${ }^{22}$ Associations have been found with the ApoE gene in European, but not in American populations. ${ }^{23}$ Genetic factors seem to be specific for defined populations and genetic findings might be restricted to certain populations.

Different genetic makeup in different populations is one possible explanation for the discordant findings in genome-wide screens in ALS. ${ }^{7,24}$ The major drawback of such studies is the need for thousands of samples from several countries to gain sufficient power. If some genetic factors are specific to certain populations, samples that are 
obtained from a mix of different cohorts might diminish the ability to detect a single susceptibility factor in any subpopulation.

\section{CONFLICT OF INTEREST}

The authors declare no conflict of interest

1 Beghi E, Logroscino G, Chio A et al: The epidemiology of ALS and the role of population-based registries. Biochim Biophys Acta 2006; 1762: 1150-1157.

2 Corcia P, Camu W, Halimi JM et al: SMN1 gene, but not SMN2, is a risk factor for sporadic ALS. Neurology 2006; 67: 1147-1150.

3 Corcia P, Mayeux-Portas V, Khoris J et al: Abnormal SMN1 gene copy number is a susceptibility factor for amyotrophic lateral sclerosis. Ann Neurol 2002. 51: 243-246.

4 Veldink JH, Kalmijn S, Van der Hout AH et al: SMN genotypes producing less SMN protein increase susceptibility to and severity of sporadic ALS. Neurology 2005; 65: 820-825.

5 Gavrilov DK, Shi X, Das K, Gilliam TC, Wang CH: Differential SMN2 expression associated with SMA severity. Nat Genet 1998; 20: 230-231.

6 Eggert C, Chari A, Laggerbauer B, Fischer U: Spinal muscular atrophy: the RNP connection. Trends Mol Med 2006; 12: 113-121.

7 Valdmanis PN, Daoud H, Dion PA, Rouleau GA: Recent advances in the genetics of amyotrophic lateral sclerosis. Curr Neurol Neurosci Rep 2009; 9: 198-205.

8 Brooks BR, Miller RG, Swash M, Munsat TL: El Escorial revisited: revised criteria for the diagnosis of amyotrophic lateral sclerosis. Amyotroph Lateral Scler Other Motor Neuron Disord 2000; 1: 293-299.

9 Echaniz-Laguna A, Guiraud-Chaumeil C, Tranchant C, Reeber A, Melki J, Warter JM: Homozygous exon 7 deletion of the SMN centromeric gene (SMN2): a potential susceptibility factor for adult-onset lower motor neuron disease. J Neurol 2002, 249: 290-293.

10 Moulard B, Salachas F, Chassande B et al: Association between centromeric deletions of the SMN gene and sporadic adult-onset lower motor neuron disease. Ann Neurol 1998; 43: 640-644.
11 Veldink JH, van den Berg LH, Cobben JM et al: Homozygous deletion of the survival motor neuron 2 gene is a prognostic factor in sporadic ALS. Neurology 2001; 56: 749-752.

12 Sleegers K, Brouwers N, Gijselinck I et al: APP duplication is sufficient to cause early onset Alzheimer's dementia with cerebral amyloid angiopathy. Brain 2006; 129: 2977-2983

13 Ibanez P, Bonnet AM, Debarges B et al: Causal relation between alpha-synuclein gene duplication and familial Parkinson's disease. Lancet 2004; 364: 1169-1171.

14 Chance PF, Fischbeck KH: Molecular genetics of Charcot-Marie-Tooth disease and related neuropathies. Hum Mol Genet 1994; 3 Spec No: 1503-1507.

15 Arkblad E, Tulinius M, Kroksmark AK, Henricsson M, Darin N: A population-based study of genotypic and phenotypic variability in children with spinal muscular atrophy. Acta Paediatr 2009; 98: 865-872.

16 Melki J, Lefebvre S, Burglen L et al: De novo and inherited deletions of the $5 \mathrm{q} 13$ region in spinal muscular atrophies. Science 1994; 264: 1474-1477

17 Atassi N, Cudkowicz ME, Schoenfeld DA: Advanced statistical methods to study the effects of gastric tube and non-invasive ventilation on functional decline and survival in amyotrophic lateral sclerosis. Amyotrophic Lateral Sclerosis 2011; 12: 272-277.

18 Andersen PM, Forsgren L, Binzer $\mathrm{M}$ et al: Autosomal recessive adult-onset amyotrophic lateral sclerosis associated with homozygosity for Asp90Ala CuZn-superoxide dismutase mutation. A clinical and genealogical study of 36 patients. Brain 1996; 119 (Part 4): 1153-1172.

19 Corcia P, Petiot P, Stevic Z et al: Respiratory onset in an ALS family with L144F SOD1 mutation. J Neurol Neurosurg Psychiatry 2011; 82: 747-749.

20 van Es MA, Dahlberg C, Birve A, Veldink JH, van den Berg LH, Andersen PM: Large-scale SOD1 mutation screening provides evidence for genetic heterogeneity in amyotrophic lateral sclerosis. J Neurol Neurosurg Psychiatry 2010; 81: 562-566.

21 Andersen PM: Amyotrophic lateral sclerosis associated with mutations in the CuZn superoxide dismutase gene. Curr Neurol Neurosci Rep 2006; 6: 37-46.

22 Greenway MJ, Andersen PM, Russ $C$ et al: ANG mutations segregate with familial and 'sporadic' amyotrophic lateral sclerosis. Nat Genet 2006; 38: 411-413.

23 Zetterberg $\mathrm{H}$, Jacobsson J, Rosengren L, Blennow K, Andersen PM: Association of APOE with age at onset of sporadic amyotrophic lateral sclerosis. J Neurol Sci 2008; 273: 67-69.

24 Garber K: Genetics. The elusive ALS genes. Science 2008; 319: 20. 